\section{Eficácia da suplementação de ferro associado ou não à vitamina $A$ no controle da anemia em escolares}

\author{
Efficacy of iron supplementation with or without \\ vitamin A for anemia control
}

\footnotetext{
${ }^{1}$ Centro de Ciências da Saúde, Universidade Federal de Pernambuco Recife, Brasil.

2 Faculdade de Ciências Médicas, Universidade de Pernambuco, Recife, Brasil. 3 Instituto Materno Infantil Professor Fernando Figueira Recife, Brasil.

Correspondência R. C. Pereira Centro de Ciências da Saúde, Universidade Federal de Pernambuco. Av. 17 de Agosto 175, apto. 601, Recife, $P E$ 52060-590, Brasil. rucape@hotlink.com.br
}

\begin{abstract}
This study aimed to evaluate the efficacy of weekly iron supplementation with or without vitamin A in the treatment of iron deficiency anemia, using an experimental, randomized, non-placebo-controlled design in 1999. 267 schoolchildren 6 to 14 years of age were randomized to two treatment groups: one group (144) received $200 \mathrm{mg}$ iron sulfate alone, with $40 \mathrm{mg}$ of elemental iron, while the other (123) received the same iron supplementation dose plus 10,000 IU of vitamin A (both groups for 30 weeks). Final anemia prevalence was reduced from $48.4 \%$ to $17.7 \%(p<0.001)$ in the group receiving iron supplementation alone and $58.1 \%$ to $14.3 \%(p<0.001)$ in the group receiving iron plus vitamin A. There was no significant difference between the groups at the end of the study according to mean $\mathrm{Hb}(p=0.355)$ and anemia $(p=0.479)$. There was a significant correction for iron deficiency anemia with weekly ironalone supplementation, but with no additional advantage of vitamin A. New studies on the synergism between these two micronutrients are recommended.
\end{abstract}

Iron-Deficiency Anemia; Vitamin A; Ferrous Sulfate; Students
Rute Cândida Pereira 1

Luiz Oscar Cardoso Ferreira 2

Alcides da Silva Diniz 1

Malaquias Batista Filho ${ }^{3}$

José Natal Figueirôa 3

\section{Introdução}

A anemia ferropriva é um importante problema de saúde pública nos países desenvolvidos e em desenvolvimento, sendo estimado que 2,3 bilhões de pessoas no mundo sejam portadoras dessa enfermidade 1 . Atualmente, no Brasil, ela se comporta com tendência temporal de ascensão 2 , sobretudo em segmentos populacionais mais vulneráveis.

A anemia compromete inúmeras funções orgânicas, tais como: o transporte de oxigênio aos tecidos, as reações de oxidação e redução, a imunidade humoral e celular, a síntese de ácido desoxirribonucléico (DNA), a síntese de neurotransmissores e, possivelmente, da mielina, dentre outras atividades vitais 3 .

As condições sócio-econômicas têm composto, com freqüência, a simultaneidade de anemia e carências nutricionais, sobretudo de ferro e de vitamina A. Dentre os fatores determinantes de anemia, o baixo nível de escolaridade materna, ausência de televisão no domicílio, falta de saneamento básico, reduzida duração do aleitamento materno exclusivo, ocorrência de diarréia, precário estado nutricional têm sido relatados como exercendo impacto significativo na redução dos níveis de hemoglobina 4.

As principais conseqüências da anemia ferropriva na criança são: retardo no crescimento e desenvolvimento motor, diminuição da atividade física, sentimento de insegurança, fadiga 
e desatenção, maior probabilidade de retardo mental 5 , alterações cognitivas e de aquisição de conceitos 6,7 , bem como baixa pontuação nos testes de rendimento mental 8 .

A carência de vitamina A acomete 127 milhões de crianças na idade pré-escolar, dentre as quais 4,4 milhões desenvolvem xeroftalmia 9, que ainda tem sido considerada como a principal causa de cegueira evitável na infância. Tendo em vista a magnitude do problema, uma série de estudos epidemiológicos, casualizados e controlados, tem destacado o importante papel da vitamina A na prevenção da morbimortalidade para algumas doenças infecciosas, a exemplo da diarréia, sarampo, malária e, provavelmente, infecção respiratória e AIDS. Uma metanálise, englobando oito ensaios clínicos, concluiu que a suplementação com vitamina A reduziu a mortalidade em cerca de $23 \%$, em crianças de seis meses a cinco anos de idade 10 .

O sinergismo do ferro e da vitamina A não está totalmente elucidado, pois é complexa a interação envolvendo múltiplas vitaminas na hematopoiese. No entanto a constatação da coexistência de carências nutricionais de ferro e vitamina A, em populações em situação de insegurança alimentar agravada por alta prevalência de anemia ferropriva, impulsionou investigações sobre o papel desses fatores nutricionais na gênese dessa anemia 11 .

$\mathrm{O}$ ferro e a vitamina A parecem agir de forma sinérgica em algumas etapas do metabolismo orgânico. É provável que a vitamina $\mathrm{A}$ atue na mobilização do ferro dos estoques dos tecidos orgânicos, favorecendo a disponibilidade do metal à hematopoiese, na síntese da hemoglobina (Hb) 11

No enfrentamento da anemia ferropriva, várias intervenções já foram testadas na prevenção e no controle desse estado carencial. O esquema intermitente do uso semanal de sais de ferro, em contraposição aos esquemas de uso diário, associado ou não à vitamina $\mathrm{A}$, tem demonstrado boa resolutividade dos quadros de anemia ferropriva 12,13 , melhoria na reposição de estoques de ferro 14,15,16 e correção da carência de vitamina A no organismo ${ }^{17}$. Além desses benefícios, no esquema semanal ocorrem relatos menos freqüentes de efeitos colaterais e maior adesão ao tratamento, com o uso do ferro na forma de sulfato ferroso heptahidratado, por administração via oral 14,18 .

O objetivo do estudo foi avaliar a eficácia da suplementação do sulfato ferroso associada ou não à vitamina A no controle da anemia ferropriva, administrados em dose semanal, nas concentrações de $\mathrm{Hb}$ e do volume corpuscular médio (VCM) de escolares.

\section{Sujeitos e método}

O estudo foi realizado no Município de São João, Estado de Pernambuco, Nordeste do Brasil. Em 1999, o município tinha 20 mil habitantes (35\% residiam na área urbana); apresentava um índice de desenvolvimento humano (IDH) de 0,593, menor que 86,9\% dos municípios do Brasil 19,20.

A população do estudo, constituída por 5.092 escolares de 39 educandários públicos, de ambos os sexos, tinha idade de 6 a 14 anos. Definiu-se como critério de inclusão a criança freqüentar regularmente a escola com assiduidade mínima de $75 \%$ do calendário escolar e de exclusão, história de hemoglobinopatias hereditárias.

O estudo foi experimental com um ensaio clínico comunitário, aleatório e cego (equipe técnica desconhecia a alocação dos sujeitos nos dois experimentos), não-controlado por placebo, realizado no período de março a dezembro de 1999. Para casualização em bloco dos escolares, segundo os dois grupos de experimentos, foram sorteadas quatro escolas mediante uso de uma tabela de números aleatórios, sendo duas rurais e duas urbanas. Todos os alunos de uma mesma escola foram alocados em um dos grupos. Um grupo recebeu $200 \mathrm{mg}$ de sulfato ferroso heptahidratado $\left(\mathrm{FeSO}_{4} \cdot 7 \mathrm{H}_{2} \mathrm{O}\right)$, contendo $40 \mathrm{mg}$ de ferro elementar, na dose de um comprimido, administrado uma vez por semana. O outro grupo recebeu a mesma dose de sulfato ferroso, associada a 10.000UI, em dose única, semanal do ingrediente ativo palmitato de retinol, marca Roche, na apresentação líquida lipossolúvel, hidromiscível.

As variáveis de mensuração do desfecho foram: concentrações de Hb e o VCM. As variáveis de controle foram: o sexo, a idade em anos completos, o peso e a estatura convertidos em escore Z, conforme as recomendações do National Center for Health Statistics (NCHS) 21. O período de suplementação foi de trinta semanas.

A amostra de 267 escolares foi estimada por meio da diferença de médias da concentração de hemoglobina de pelo menos 0,5g/dL, com probabilidade do erro tipo I igual a $5 \%$, um poder de $90 \%$ (1- $\beta$ ) e uma variância de $1,3 \mathrm{~g} / \mathrm{dL}$, pela fórmula matemática 22 baseada nos dados de Lopes et al. 14, obtendo-se tamanho amostral de 109, que foi acrescido de 30\%, devido à evasão escolar.

Antecedendo às suplementações, foi administrado albendazol, 400mg em dose única, para todos os escolares, independentemente de estarem parasitados. $\mathrm{O}$ experimento foi desenvolvido simultaneamente às atividades escolares. Uma funcionária de nível superior do Departamento de Vigilância Sanitária do município, responsável pela supervisão dos programas desenvolvidos 
nas escolas, mensalmente, entregava aos professores regentes de classe, depois de capacitados, o total de doses dos alunos de suas turmas. Estes foram responsáveis pela administração e registro da suplementação dos micronutrientes em formulário próprio que, no mês subseqüente, foi entregue à funcionária supervisora a quem coube, após conferir os registros de administração, fornecer as novas doses mensais. Os relatórios mensais de administração foram encaminhados aos pesquisadores que, durante todo o experimento, realizaram supervisão direta. Não foram necessárias correções de condutas ao longo das trinta semanas de suplementação.

Aproximadamente $5 \mathrm{~mL}$ de sangue foram colhidos, por punção venosa periférica, com seringa e agulha descartáveis, utilizando como anticoagulante o ácido etileno-diamino-tetracético (EDTA). A Hb e o VCM foram analisados em contador de múltiplos canais, marca Coulter, modelo T890 (Coulter Electronics). Tomando-se como parâmetros a Hb e o VCM, os escolares foram categorizados, respectivamente, em anêmi$\cos (\mathrm{Hb}<12 \mathrm{~g} / \mathrm{dL})$ e portadores de microcitose (VCM $<80 \mathrm{fL}$ ).

Mensuraram-se o peso e a estatura dos escolares, usando vestuário mínimo, sem adornos na cabeça e sem calçados, em balança digital, com sensibilidade de $100 \mathrm{~g}$ e capacidade de $100 \mathrm{~kg}$ e em antropômetro de parede, com precisão de $0,1 \mathrm{~cm}$. Foram adotados os índices antropométricos peso para a idade e altura para idade, em escores $\mathrm{Z}( \pm 2 \mathrm{DP})$.

As análises dos dados foram efetuadas com os programas Epi Info, versão 6.04d (Centers for Disease Control and Prevention, Atlanta, Estados Unidos) e StatXact 3.0 (Cytel Inc., Cambridge, Estados Unidos).

A descrição estatística foi realizada com base em medidas de posição e de dispersão. Foram utilizados os testes: t de Student para comparação de duas médias; qui-quadrado para testar a associação entre duas variáveis categóricas e o teste de McNemar para comparar proporções pareadas. O nível de significância adotado foi $5 \%$.

O estudo foi aprovado pelo Comitê de Ética em Pesquisa do Instituto Materno Infantil Professor Fernando Figueira (IMIP), e a participação das crianças foi autorizada pelos pais ou responsáveis, segundo Resolução no . 196/96 do Ministério da Saúde.

\section{Resultados}

Dos 267 escolares, verificou-se que mais da metade $(53,2 \%)$ era portadora de anemia, embora apenas $11,2 \%$ tivessem microcitose. Todavia, $83,3 \%$ dos escolares que apresentaram microcitose tinham simultaneamente anemia.

O estado nutricional, avaliado pela relação do peso para idade, mostrou que 7,1\% (19/267) dos escolares tinham baixo peso. Não se observou existência de sobrepeso ou obesidade. $\mathrm{O}$ indicador estatura para idade mostrou que $11,2 \%$ (30/267) dos escolares apresentavam retardo do crescimento linear.

Por um lado, não foram observadas diferenças estatísticas significantes das características hematológicas, demográficas e antropométricas, antes da suplementação, entre os dois grupos experimentais (Tabela 1).

Por outro lado, as análises das características hematológicas ( $\mathrm{Hb}$ e VCM), demográficas (idade e sexo) e do estado nutricional (baixo peso e baixa estatura para a idade), antes da suplementação, não mostraram diferença estatisticamente significante entre o grupo de perdas e de remanescentes alocados no estudo (Tabela 2). Em relação à alocação dos escolares no grupo suple-

Tabela 1

Comparabilidade entre os grupos de estudo, segundo as variáveis hematológicas, demográficas e antropométricas, antes da suplementação. Município de São João, Pernambuco, Brasil, 1999.

\begin{tabular}{|c|c|c|c|}
\hline Características & Sulfato ferroso & Sulfato ferroso + vitamina A & $p$ \\
\hline $\mathrm{Hb}(\mathrm{g} / \mathrm{dL})[$ média $\pm \mathrm{DP}]$ & $12,0 \pm 1,0$ & $11,8 \pm 0,9$ & 0,335 \\
\hline VCM (fL) [média \pm DP] & $85,3 \pm 5,3$ & $86,3 \pm 5,0$ & 0,131 \\
\hline Idade (anos) [média \pm DP] & $9,8 \pm 2,2$ & $9,5 \pm 2,3$ & 0,379 \\
\hline Sexo masculino [\%] & 52,1 & 48,0 & 0,503 \\
\hline Peso/ldade [\% baixo peso] & 5,6 & 8,9 & 0,283 \\
\hline Altura/ldade [\% baixa estatura] & 9,0 & 13,8 & 0,251 \\
\hline
\end{tabular}

DP: desvio padrão; Hb: hemoglobina; VCM: volume corpuscular médio. 
Características hematológicas, demográficas e antropométricas das perdas e dos remanescentes alocados no estudo. Município de São João, Pernambuco, Brasil, 1999.

\begin{tabular}{lccc}
\hline Características & $\begin{array}{c}\text { Perdas } \\
(\mathbf{n}=\mathbf{3 8})\end{array}$ & $\begin{array}{c}\text { Remanescentes } \\
(\mathbf{n}=\mathbf{2 2 9})\end{array}$ \\
\hline $\mathrm{Hb}(\mathrm{g} / \mathrm{dL})$ [média $\pm \mathrm{DP}]$ & $12,0 \pm 0,9$ & $11,9 \pm 1,0$ & 0,626 \\
VCM (fL) [média $\pm \mathrm{DP}]$ & $86,1 \pm 5,2$ & $85,7 \pm 5,1$ & 0,636 \\
Idade (anos) [média $\pm \mathrm{DP}]$ & $10,1 \pm 2,5$ & $9,6 \pm 2,2$ & 0,170 \\
Sexo masculino [\%] & 39,5 & 52,0 & 0,153 \\
Peso/ldade [\% baixo peso] & 7,9 & 11,8 & 0,246 \\
Altura/ldade [\% baixa estatura] & 2,6 & 7,9 & 0,713 \\
\hline
\end{tabular}

DP: desvio padrão; Hb: hemoglobina; VCM: volume corpuscular médio.

mentado com sulfato ferroso, iniciou-se com 144 e terminou com 124 escolares, correspondendo a uma perda de 13,9\%; no grupo sulfato ferroso associado à vitamina A, teve início com 123 e terminou com 105 escolares, ou seja, com uma perda de $14,2 \%$. Entretanto não se observou diferença estatística significante $(p=0,862)$ entre os dois esquemas e as perdas de seguimento.

\section{Eficácia das suplementações}

Dentre os escolares que receberam o sulfato ferroso exclusivamente, houve incremento nas médias das concentrações de $\mathrm{Hb}$ de $0,9 \pm 0,9 \mathrm{~g} / \mathrm{dL}$ ( $\mathrm{p}<0,001)$ e aumento significante do VCM de $1,6 \pm 4,5 \mathrm{fL}(\mathrm{p}<0,001)$. Antes da suplementação exclusiva do SF, a proporção de escolares anêmicos foi $48,4 \%$, sofrendo redução significante para $17,7 \%$ depois do experimento ( $p<0,001$ ) dos casos de anemia entre os escolares. Não foi, contudo, evidenciado qualquer impacto significante na redução da prevalência de microcitose $(\mathrm{p}=$ $0,388)$. Concernente ao grupo de escolares que recebeu sulfato ferroso associado à vitamina $\mathrm{A}$, ocorreram incremento estatisticamente significante nas médias das concentrações de $\mathrm{Hb}$ de 0,8 $\pm 0,8 \mathrm{~g} / \mathrm{dL}(\mathrm{p}<0,001)$ e aumento estatisticamente significante do VCM de $1,4 \pm 1,9 \mathrm{fL}(\mathrm{p}<0,001)$. Antes da suplementação do sulfato ferroso associado à vitamina $\mathrm{A}$, observou-se que a proporção de escolares anêmicos foi $58,1 \%$, reduzindo-se significantemente para $14,3 \%$ depois do experimento $(\mathrm{p}<0,001)$. A suplementação combinada não evidenciou, porém, impacto significante na redução da prevalência de microcitose $(\mathrm{p}=$ 0,388 ). Na comparação intergrupos, verificou-se não ter havido diferença estatisticamente significante na efetividade das suplementações com sulfato ferroso exclusivamente ou associado à vitamina A sobre os parâmetros hematológicos de Hb ( $p=0,355)$ e VCM ( $p=0,673)$, bem como anemia $(p=0,479)$ e microcitose $(p=0,449)$ (Tabela 3$)$.

\section{Discussão}

A prevalência de anemia dentre os escolares estudados foi alta, uma vez que mais da metade deles apresentou concentrações de hemoglobina inferiores a $12 \mathrm{~g} / \mathrm{dL}$. Esses resultados seriam presumíveis, considerando as precárias condições sócio-econômicas do município, consubstanciadas em um IDH de 0,593 e agravadas pela situação crítica das condições de moradia, da escassez de água potável e de saneamento básico 19.

A anemia microcítica alcançou, paradoxalmente, baixo percentual de ocorrência pressupondo-se, dessa forma, que essa enfermidade seja menos prevalente do que se esperava nesse contexto ecológico, mas esta hipótese estaria em "princípio" afastada, considerando-se o impacto significante da suplementação com ferro na correção da anemia, observada neste estudo. Uma segunda hipótese de maior plausibilidade seria a de que as alterações morfológicas sofridas pelo eritrócito parecem ocorrer em um estágio da hematopoiese bem posterior ao decréscimo nas concentrações de $\mathrm{Hb}{ }^{1}$.

As pesquisas têm avaliado a eficácia das estratégias de intervenção utilizadas no enfrentamento da anemia ferropriva. Dentre as ações propostas, destacam-se a administração semanal de sais de ferro, assim como a suplementação do ferro associada a outros micronutrientes como formas de intervenção para prevenção e controle desse estado carencial. 
Concentrações de hemoglobina (Hb), volume corpuscular médio (VCM), anemia e microcitose pós-suplementação com sulfato ferroso exclusivamente ou associado à vitamina A em escolares. Município de São João, Pernambuco, Brasil, 1999.

\begin{tabular}{|c|c|c|c|c|c|c|c|}
\hline \multirow[t]{3}{*}{ Parâmetros } & \multicolumn{6}{|c|}{ Suplementação } & \multirow[t]{3}{*}{$p^{\star *}$} \\
\hline & \multicolumn{3}{|c|}{ Sulfato ferroso } & \multicolumn{3}{|c|}{ Sulfato ferroso + vitamina A } & \\
\hline & $\begin{array}{c}\text { Antes } \\
(n=144)\end{array}$ & $\begin{array}{c}\text { Após } \\
(\mathrm{n}=124)\end{array}$ & $p$ * & $\begin{array}{c}\text { Antes } \\
(n=123)\end{array}$ & $\begin{array}{c}\text { Após } \\
(\mathrm{n}=105)\end{array}$ & $p$ * & \\
\hline \multicolumn{8}{|l|}{$\mathrm{Hb}$} \\
\hline Média & 11,9 & 12,8 & $<0,001$ & 11,8 & 12,6 & $<0,001$ & 0,355 \\
\hline Desvio-padrão & 1,0 & 0,9 & & 0,9 & 0,8 & & \\
\hline \multicolumn{8}{|l|}{ VCM } \\
\hline Média & 85,3 & 86,9 & $<0,001$ & 86,2 & 87,6 & $<0,001$ & 0,673 \\
\hline Desvio-padrão & 5,4 & 5,5 & & 4,8 & 5,0 & & \\
\hline Anemia (\%) & 48,4 & 17,7 & $<0,001 * \star \star$ & 58,1 & 14,3 & $<0,001 * \star \star$ & $0,479 \#$ \\
\hline Microcitose (\%) & 12,1 & 8,9 & $0,388 * \star \star$ & 10,5 & 6,7 & $0,388 * \star \star$ & $0,449 \#$ \\
\hline
\end{tabular}

* Teste t de Student para amostras pareadas;

** Teste $\mathrm{t}$ de Student para comparação intergrupos;

*** Teste de McNemar;

\# Teste qui-quadrado.

A eficácia da suplementação semanal com sulfato ferroso isoladamente foi elevada, uma vez que o incremento substancial nas médias das concentrações de hemoglobina foi da ordem de $0,9 \mathrm{~g} / \mathrm{dL}$. Mas a ausência de impacto no volume corpuscular médio poderia ser explicada pela baixa prevalência de microcitose observada.

Estudos publicados apresentam resultados da correção de anemia semelhantes aos aqui observados, utilizando esquema semanal de aporte de ferro 23 . Outros efeitos promissores são relatados, a exemplo do incremento significante da concentração média de $\mathrm{Hb}$ 12,24, e queda da prevalência de anemia 4,12. Esses resultados consolidam cada vez mais o sucesso da eficácia com os esquemas semanais de suplementação de ferro.

Nos resultados aqui apresentados, embora a suplementação combinada tenha mostrado um impacto significante, tanto na correção da anemia, quanto no incremento da concentração média de $\mathrm{Hb}$, não foi evidenciado qualquer efeito terapêutico adicional dessa suplementação, comparada com a suplementação isolada de ferro. Contudo, Angeles-Agdeppa et al. 25 identificaram que a suplementação semanal com ferro e vitamina A aumentou o status do mineral em adolescentes.

Algumas hipóteses poderiam ser aventadas para uma explicação plausível da ausência do efeito na suplementação combinada observado neste estudo. Uma primeira seria atribuir ao fato de que o status orgânico de vitamina A poderia ser adequado na população pesquisada, ainda que no contexto ecológico estudado sejam muitos os fatores de risco para a deficiência de micronutrientes, inclusive da vitamina A 19. Entretanto, uma prevalência baixa de deficiência de vitamina $A$, entre os escolares de São João, seria esperada, considerando que o programa de combate à hipovitaminose $\mathrm{A}$, desenvolvido pelos ministérios da Saúde e da Educação, no seu espectro de ações, tem englobado a distribuição de megadoses de vitamina A, aumento da cobertura vacinal, bem como reforço na qualidade da merenda escolar na área do estudo.

Por outro lado, admitindo-se a hipótese reversa, na qual a população apresentaria carência de vitamina A, a dose utilizada na suplementação (10.000UI/semanal) não teria sido suficiente para repor as necessidades orgânicas e provocar um impacto mensurável sobre o status do ferro, com efeitos significativos na eritropoiese. Vale ainda ressaltar que a ocorrência simultânea da carência de outros nutrientes, a exemplo do zinco, poderia igualmente atuar inibindo a ação interativa da vitamina A.

Segundo Semba \& Bloem 11, poucos estudos relatam a percentagem de indivíduos com deficiência de vitamina $\mathrm{A}$ associada à anemia. No entanto esses autores afirmam que a prevalência da anemia é alta em populações afetadas por deficiência de vitamina A, em países em desenvolvimento, e que a melhoria do status de vitamina 
A parece contribuir para a redução da anemia 11. Nesse sentido, Diniz ${ }^{26}$, estudando pré-escolares da Paraíba, no Nordeste do Brasil, observou em um modelo de regressão múltipla que, para cada unidade de mudança no retinol sérico, houve uma variação positiva de 0,11 unidades de $\mathrm{Hb}$.

Os resultados observados no nosso estudo apresentam indícios da eficácia do esquema com sulfato ferroso exclusivamente na preven-

\section{Resumo}

Com o objetivo de avaliar a eficácia da suplementação de ferro, associado ou não à vitamina A, na anemia ferropriva, administrado semanalmente, realizou-se ensaio clínico comunitário, randomizado, não controlado por placebo, em 1999. Uma amostra probabilística de 267 escolares de ambos os sexos com 6 a 14 anos de idade foram casualizados em bloco segundo dois tipos de intervenção: um grupo (144) recebeu $200 \mathrm{mg}$ de sulfato ferroso com (40mg de ferro elementar) $e o$ outro (123) recebeu dose similar de sulfato ferroso associado a 10.000 UI de vitamina A, durante 30 semanas. A prevalência de anemia ao final foi reduzida de $48,4 \%$, para $17,7 \%$ ( $p<0,001)$, no grupo que recebeu sulfato ferroso, e de 58,1\%, para 14,3\% ( $p<0,001)$, no grupo que recebeu sulfato ferroso associado à vitamina A. Não houve diferença significante entre os grupos, ao final do estudo, quanto às médias de $\mathrm{Hb}(p=0,355)$ e à proporção de anêmicos $(p=0,479)$. Corrigiu-se significantemente a anemia ferropriva com suplementação semanal de sulfato ferroso, mas não houve vantagem adicional com a associação da vitamina A. Sugerem-se novos estudos sobre o sinergismo desses micronutrientes.

Anemia Ferropriva; Vitamina A; Sulfato Ferroso; Estudantes ção e controle da deficiência de ferro e da anemia em escolares, no entanto parece não haver um efeito sinérgico potencializador da resposta hematológica com a vitamina A, na suplementação combinada com ferro. Sugerem-se novos estudos sobre o sinergismo desse micronutriente no status do ferro, inclusive com as mensurações de sua dosagem hematológica antes e depois das suplementações.

\section{Colaboradores}

Todos os autores participaram da elaboração do artigo, definindo os critérios metodológicos, a estrutura e o conteúdo. R. C. Pereira e L. O. C. Ferreira foram responsáveis pela coleta de dados e redação do texto, que foi criticado e revisto diversas vezes por A. S. Diniz e M. Batista Filho. O tratamento estatístico dos dados foi realizado por R. C. Pereira e L. O. C. Ferreira com auxílio de J. N. Figueirôa. Todos os autores foram responsáveis pelo levantamento bibliográfico.

\section{Agradecimentos}

O presente artigo utilizou parte dos dados da Tese de Doutorado desenvolvida em projeto de parceria interinstitucional (Instituto Nacional de Alimentação e Nutrição, Ministério da Saúde/Instituto Materno Infantil Professor Fernando Figueira/Universidade Federal de Pernambuco/Universidade de Pernambuco), apresentada ao Programa de Pós-graduação em Nutrição da Universidade Federal de Pernambuco. 


\section{Referências}

1. World Health Organization/United Nations Children Fund/United Nations University. Iron deficiency anemia assessment, prevention, and control. A guide for program managers. Geneva: World Health Organization; 2001.

2. Batista Filho M, Rissin A. A transição nutricional no Brasil: tendências regionais e temporais. Cad Saúde Pública 2003; 19 Suppl 1:S181-91.

3. Olivares MG, Tomás WK. Consecuencias de la deficiencia de hierro. Rev Chil Nutr 2003; 30:226-33.

4. Lima ACVMS, Lira PIC, Romani SAM, Eickmann SH, Lima, MC. Fatores determinantes dos níveis de hemoglobina em crianças aos 12 meses de vida na Zona da Mata Meridional de Pernambuco. Rev Bras Saúde Matern Infant 2004; 4:35-43.

5. Hurtado EK, Claussen AH, Scott KG. Early childhood anemia and mild or moderate mental retardation. Am J Clin Nutr 1999; 69:115-9.

6. Soewondo S. The effect of iron deficiency and mental stimulation on Indonesian children's cognitive performance and development. Kobe J Med Sci 1995; 41:1-17.

7. Rivera AF, Walter KT. Efecto de la anemia ferropriva en el lactante sobre el desarrollo psicológico del escolar. J Pediatr (Rio J) 1997; 73 Suppl 1:S49.

8. Lozoff B, Wolf AW, Jimenez E. Iron-deficiency anemia and infant development: effects of extended oral iron therapy. J Pediatr 1996; 129:382-9.

9. West Jr. KP. Extent of vitamin A deficiency among preschool children and women of reproductive age. J Nutr 2002; 132:28575-665.

10. Beaton GH, Martorell R, Aronson KA, Edmonston B, McCabe G, Ross AC, et al. La suplementación con vitamina A y la morbilidad y mortalidad infantil en los países en desarrollo. Bol Oficina Sanit Panam 1994; 117:506-18.

11. Semba RD, Bloem MW. The anemia of vitamin A deficiency: epidemiology and pathogenesis. Eur J Clin Nutr 2002; 56:271-81.

12. Brunken GS, Muniz PT, Silva SM. Suplementação semanal com sulfato ferroso reduz em $1 / 3$ a prevalência de anemia em pré-escolares. Rev Bras Epidemiol 2004; 7:210-9.

13. Ferreira LOC. A ação do sulfato ferroso administrado em doses diárias e semanais em escolares da mata sul de Pernambuco: um ensaio terapêutico [Tese de Doutorado]. Recife: Departamento de Nutrição, Universidade Federal de Pernambuco; 1998.

14. Lopes MCS, Ferreira LOC, Batista Filho M. Uso diário e semanal de sulfato ferroso no tratamento de anemia em mulheres no período reprodutivo. Cad Saúde Pública 1999; 15:799-808.
15. Beasley NM, Tomkins AM, Hall A, Lorri W, Kihamia $\mathrm{CM}$, Bundy DA. The impact of weekly iron supplementation on the iron status and growth of adolescent girls in Tanzania. Trop Med Int Health 2000; 5:794-9.

16. Liu X, Yang W, Song Y. Evaluation of the effects of intermittent iron supplement on iron-deficiency anemia in children. Zhonghua Yu Fang Yi Xue Za Zhi 1995; 29:34-7.

17. Ahmed F, Khan MR, Jackson AA. Concomitant supplemental vitamin A enhances the response to weekly supplemental iron and folic acid in anemic teenagers in urban Bangladesh. Am J Clin Nutr 2001; 74:108-15.

18. Souza AI, Batista Filho M, Ferreira, LOC, Figueirôa JN. Efetividade de três esquemas com sulfato ferroso para tratamento de anemia em gestantes. Rev Panam Salud Pública 2004; 15:313-9.

19. Instituto de Planejamento de Pernambuco. Pernambuco em dados. Recife: Instituto de Planejamento de Pernambuco; 2000.

20. Agência Estadual de Planejamento e Pesquisas de Pernambuco. Perfil municipal; informações municipais - 1999. Recife: Agência Estadual de Planejamento e Pesquisas de Pernambuco; 2000.

21. National Center for Health Statistics. Growth curves for children birth-18 years. Washington DC: National Center for Health Statistics; 1977.

22. Kirkwood BR. Essentials of medical statistics. London: Blackwell Scientific Publications; 1988.

23. Hall A, Roschnik N, Ouattara F, Toure I, Maiga F, Sacko M, et al. A randomized trial in Mali of the effectiveness of weekly iron supplements given by teachers on the haemoglobin concentrations of schoolchildren. Public Health Nutr 2002; 5:413-6.

24. Monteiro CA, Szarfarc SC, Brunken GS, Gross R, Conde WL. A prescrição semanal do sulfato ferroso pode ser altamente efetiva para reduzir níveis endêmicos de anemia na infância. Rev Bras Epidemiol 2002; 5:71-80.

25. Angeles-Agdeppa I, Schultink W, Sastroamidjojo S, Gross R, Karyadi D. Weekly micronutrient supplementation to build iron stores in female Indonesian adolescents. Am J Clin Nutr 1997; 66:177-83.

26. Diniz AS. Combate à deficiência de vitamina A: linhas de ação e perspectivas. Rev Bras Saúde Matern Infant 2001; 1:31-6.

Recebido em 16/Mar/2006

Versão final reapresentada em 28/Jul/2006

Aprovado em 20/Dez/2006 